\title{
A Kind of Infinite-Dimensional Novikov Algebras and Its Realizations
}

\author{
Liangyun Chen \\ School of Mathematics and Statistics, Northeast Normal University, Changchun 130024, China \\ Correspondence should be addressed to Liangyun Chen; chenly640@nenu.edu.cn
}

Received 24 May 2013; Accepted 16 July 2013

Academic Editor: T. Raja Sekhar

Copyright (C) 2013 Liangyun Chen. This is an open access article distributed under the Creative Commons Attribution License, which permits unrestricted use, distribution, and reproduction in any medium, provided the original work is properly cited.

We construct a kind of infinite-dimensional Novikov algebras and give its realization by hyperbolic sine functions and hyperbolic cosine functions.

\section{Introduction}

Novikov algebras were introduced in connection with Hamiltonian operators in the formal variational calculus and the Poisson brackets of hydrodynamic type. They were used to construct the Virasoro-type Lie algebras. So the study of Novikov algebras is interesting in both mathematics and mathematical physics.

When Gel'fand and Diki [1,2] and Gel'fand and Dorfman [3] studied the following operator:

$$
H_{i j}=\sum_{k} c_{i j k} u_{k}^{(1)}+d_{i j k} u_{k}^{(0)} \frac{\mathrm{d}}{\mathrm{d} x}, \quad c_{i j k} \in \mathbb{C}, d_{i j k}=c_{i j k}+c_{j i k},
$$

they gave the definition of Novikov algebras. Concretely, let $c_{i j k}$ be the structural coefficients, and let a product of $L=$ $L\left(e_{0}, e_{1}, \ldots\right)$ be o such that

$$
e_{i} \circ e_{j}=\sum c_{i j k} e_{k} .
$$

For any $a, b, c \in L$, the product is Hamilton operator if and only if o satisfies

$$
\begin{gathered}
(a \circ b) \circ c=(a \circ c) \circ b, \\
(a \circ b) \circ c+c \circ(a \circ b)=(c \circ b) \circ a+a \circ(c \circ b) .
\end{gathered}
$$

Ma presented many new soliton hierarchies of commuting bi-Hamiltonian evolution equations from the so-called Novikov algebras [4-6]. In 1987, Zel'manov [7] began to study Novikov algebras and proved that the dimension of finite-dimensional simple Novikov algebras over a field of characteristic zero is one. In algebras, what are paid attention to by mathematician are classifications and structures, but so far we have not got the systematic theory for general Novikov algebras. In 1992, Osborn [8-10] had finished the classification of infinite simple Novikov algebras with nilpotent elements over a field of characteristic zero and finite simple Novikov algebras with nilpotent elements over a field of characteristic $p>0$. In 1995, Xu [10-13] developed his theory and got the classification of simple Novikov algebras over an algebraically closed field of characteristic zero. Bai and Meng [14-16] did a series of researches on low dimensional Novikov algebras, such as the structure and classification. We construct two kinds of Novikov algebras [17]. Recently, people obtained some properties in Novikov superalgebras [18, 19]. In this paper, we construct an infinite-dimensional Novikov algebra and give its realization by hyperbolic sine functions and hyperbolic cosine functions.

Definition 1 (see [17]). Let $(\mathscr{A}, \circ)$ be an algebra over $\mathbf{F}$ such that

$$
\begin{gathered}
a \circ(b \circ c)-(a \circ b) \circ c=b \circ(a \circ c)-(b \circ a) \circ c, \\
(a \circ b) \circ c=(a \circ c) \circ b, \quad \forall a, b, c \in \mathscr{A},
\end{gathered}
$$

and then $\mathscr{A}$ is called a Novikov algebra over $\mathbf{F}$.

Remark 2. An algebra $\mathscr{A}$ is called a left symmetric algebra if it only satisfies (4). It is clear that left symmetric algebras contain Novikov algebras. 
Remark 3. (1) If $(\mathscr{A}, \circ)$ is a left symmetric algebra satisfying

$$
[a, b]=a \circ b-b \circ a, \quad \forall a, b \in \mathscr{A},
$$

then $(\mathscr{A},[]$,$) is a Lie algebra. Usually, it is called an adjoining$ Lie algebra.

(2) Let $(\mathscr{A}, \cdot)$ be a commutative algebra, and then $(\mathscr{A}$, $\left.d_{0}, \circ\right)$ is a Novikov algebra if $d_{0}$ is a derivation of $\mathscr{A}$ with a bilinear operator $\circ$ such that

$$
a \circ b=a \cdot d_{0}(b), \quad \forall a, b \in \mathscr{A} .
$$

\section{Main Results}

Lemma 4. Let $\left\{b_{0}, a_{1}, b_{1}, a_{2}, b_{2}, \ldots a_{n}, b_{n}, \ldots\right\}$ be a basis of the linear space $\mathscr{A}$ over a field $\mathbf{F}$ of characteristic $p \neq 2$ satisfying

$$
\begin{gathered}
a_{m} a_{n}=\frac{1}{2}\left(b_{m+n}-b_{m-n}\right), \\
b_{m} b_{n}=\frac{1}{2}\left(b_{m+n}+b_{m-n}\right), \\
a_{m} b_{n}=b_{n} a_{m}=\frac{1}{2}\left(a_{m+n}+a_{m-n}\right),
\end{gathered}
$$

where $b_{-m}=b_{m}, a_{-m}=-a_{m}$. Then $\mathscr{A}$ is a commutative and associative algebra.

Proof. It is clear that $\mathscr{A}$ is a commutative algebra over F:

$$
\begin{aligned}
& \left(a_{k}, a_{n}, a_{m}\right) \\
& =a_{k}\left(a_{n} a_{m}\right)-\left(a_{k} a_{n}\right) a_{m} \\
& =\frac{1}{2} a_{k}\left(b_{m+n}-b_{n-m}\right)-\frac{1}{2}\left(b_{k+n}-b_{k-n}\right) a_{m} \\
& =\frac{1}{4}\left(a_{k+m+n}+a_{k-m-n}-a_{k+n-m}-a_{k-n+m}\right. \\
& \left.\quad-a_{m+k+n}-a_{m-k-n}+a_{m+k-n}+a_{m-k+n}\right)
\end{aligned}
$$$$
=0 .
$$

Similarly, we have that $\left(b_{k}, b_{n}, b_{m}\right)=\left(a_{k}, a_{n}, b_{m}\right)=\left(a_{k}, b_{n}\right.$, $\left.a_{m}\right)=\left(b_{k}, a_{n}, a_{m}\right)=\left(b_{k}, b_{n}, a_{m}\right)=\left(b_{k}, a_{n}, b_{m}\right)=\left(a_{k}, b_{n}, b_{m}\right)=$ 0 . Then $(a, b, c)=0, \forall a, b, c \in \mathscr{A}$. The result follows.

Corollary 5. $b_{0}$ of Lemma 4 is a unity of $\mathscr{A}$.

Lemma 6. Let $\mathscr{A}$ be a commutative and associative algebra satisfying Lemma 4. Then the following statements hold:

(1) If $D_{0}$ is a linear transformation of $\mathscr{A}$ such that

$$
\begin{aligned}
& D_{0}\left(a_{n}\right)=n b_{n}, \quad n=1,2,3, \ldots, \\
& D_{0}\left(b_{n}\right)=n a_{n}, \quad n=0,1,2, \ldots,
\end{aligned}
$$

then $D_{0}$ is a derivation of $\mathscr{A}$.

(2) If $a D_{0}$ is a linear transformation of $\mathscr{A}$ such that

$$
\left(a D_{0}\right)(b)=a D_{0}(b), \quad \forall a, b \in \mathscr{A},
$$

then $a D_{0}$ is a derivation of $\mathscr{A}$.
(3) $\mathscr{D}_{1}=\left\{a D_{0} \mid a \in \mathscr{A}\right\}$ is a subalgebra of Lie algebra $\operatorname{Der} \mathscr{A}$.

Proof. (1) We have

$$
\begin{aligned}
D_{0}\left(a_{n} a_{m}\right)= & D_{0}\left(\frac{1}{2}\left(b_{n+m}-b_{n-m}\right)\right) \\
= & \frac{1}{2}\left((m+n) a_{n+m}-(n-m) a_{n-m}\right), \\
D_{0}\left(a_{n}\right) a_{m}+a_{n} D_{0}\left(a_{m}\right)= & n b_{n} a_{m}+m a_{n} b_{m} \\
= & \frac{n}{2}\left(a_{n+m}-a_{n-m}\right) \\
& +\frac{m}{2}\left(a_{n+m}-a_{m-n}\right) \\
= & \frac{1}{2}\left((m+n) a_{m+n}-(n-m) a_{n-m}\right) .
\end{aligned}
$$

So $D_{0}$ is a derivation of $\mathscr{A}$.

(2) For $\forall a, b, c \in \mathscr{A}$, we have

$$
\begin{aligned}
\left(a D_{0}\right)(b c) & =a D_{0}(b c)=a D_{0}(b) c+a b D_{0}(c) \\
& =\left(a D_{0}\right)(b) c+b\left(a D_{0}\right)(c)
\end{aligned}
$$

so $a D_{0}$ is a derivation of $\mathscr{A}$.

(3) For $\forall a, b, c \in \mathscr{A}$, we have

$$
\begin{aligned}
{\left[a D_{0}, b D_{0}\right](c) } & =\left(a D_{0}\right)\left(b D_{0}\right)(c)-\left(b D_{0}\right)\left(a D_{0}\right)(c) \\
& =a D_{0}(b) D_{0}(c)-b D_{0}(a) D_{0}(c) \\
& =\left(a D_{0}(b)-b D_{0}(a)\right) D_{0}(c) .
\end{aligned}
$$

Then $\left[a D_{0}, b D_{0}\right]=\left(a D_{0}(b)-b D_{0}(a)\right) D_{0} \in \mathscr{D}_{1}$, and so (3) holds.

Theorem 7. Let $\mathscr{A}$ be a commutative and associative algebra satisfying Lemma 4 , and let a be an element of $\mathscr{A}$. If $D_{0}$ satisfies Lemma 6 and o satisfies

$$
b \circ c=b a D_{0}(c), \quad \forall b, c \in \mathscr{A},
$$

then the following statements hold:

(1) $\left(\mathscr{A}, a D_{0}, \circ\right)$ is a Novikov algebra.

(2) $\left(\mathscr{A}, a D_{0},[],\right)$ is an adjoining Lie algebra of $\left(\mathscr{A}, a D_{0}, \circ\right)$ and $[$,$] such that$

$$
[b, c]=a\left(b D_{0}(c)-c D_{0}(b)\right), \quad \forall b, c \in \mathscr{A} .
$$

Proof. (1) By Lemma 6, $a D_{0}$ is a derivation of the commutative algebra $\mathscr{A}$. So $\left(\mathscr{A}, a D_{0}, \circ\right)$ is a Novikov algebra by Remark 3(2).

(2) $\left(\mathscr{A}, a D_{0},[],\right)$ is an adjoining Lie algebra of $\left(\mathscr{A}, a D_{0}, \circ\right)$ by Remark 3(1). For $\forall b, c \in \mathscr{A}, \exists a \in \mathscr{A}$, we have

$$
\begin{aligned}
{[b, c] } & =b \circ c-c \circ b \\
& =b a D_{0}(c)-c a D_{0}(b)=a\left(b D_{0}(c)-c D_{0}(b)\right)
\end{aligned}
$$

since $\mathscr{A}$ is commutative. Hence we obtain the desired result. 
Let $b_{0}$ be a unity of $\mathscr{A}$. If we set $a=b_{0}$ in Theorem 7, then $a_{n} \circ a_{m}=a_{n} b_{0} D_{0}\left(a_{m}\right)=a_{n}\left(m b_{m}\right)=(m / 2)\left(a_{m+n}+a_{n-m}\right)$. Similarly, we obtain the following corollary.

Corollary 8. Let $\mathscr{A}$ be a commutative and associative algebra satisfying Lemma 4 . Then the following statements hold:

$$
\begin{gathered}
a_{n} \circ a_{m}=\frac{m}{2}\left(a_{n+m}+a_{n-m}\right), \\
b_{n} \circ b_{m}=\frac{m}{2}\left(a_{n+m}+a_{m-n}\right), \\
a_{n} \circ b_{m}=\frac{m}{2}\left(b_{n+m}-b_{n-m}\right), \\
b_{n} \circ a_{m}=\frac{m}{2}\left(b_{n+m}+b_{n-m}\right), \\
{\left[a_{n}, a_{m}\right]=\frac{1}{2}(m-n) a_{n+m}+\frac{1}{2}(m+n) a_{n-m},} \\
{\left[b_{n}, b_{m}\right]=\frac{1}{2}(m-n) a_{n+m}-\frac{1}{2}(m+n) a_{n-m},} \\
{\left[a_{n}, b_{m}\right]=\frac{1}{2}(m-n) b_{n+m}-\frac{1}{2}(n+m) b_{n-m},} \\
{\left[b_{n}, a_{m}\right]=\frac{1}{2}(m-n) b_{n+m}+\frac{1}{2}(m+n) b_{n-m} .}
\end{gathered}
$$

We have the following: let $\sinh x=\left(e^{x}-e^{-x}\right) / 2, \cosh x=$ $\left(e^{x}+e^{-x}\right) / 2$, and let the field $\mathbf{F}$ be assumed $\mathbf{R}$ or $\mathbf{C}$. We will construct Novikov algebras over the linear space which is generated by $\sinh x$ and $\cosh x$.

First, let $\mathscr{T}$ be a linear space generated by $\{\sinh m x, \cosh n x \mid m, n \in \mathbf{N}\}$ over $\mathbf{F}$.

Lemma 9. $\mathscr{T}$ satisfying the above product is a commutative associative algebra.

Proof. Since the above product is commutative and associative, we only need $\mathscr{T}$ to be closed for the product. In fact,

$\sinh m x \sinh n x=\frac{1}{2}[\cosh (m+n) x-\cosh (m-n) x]$,

$\cosh m x \cosh n x=\frac{1}{2}[\cosh (m+n) x+\cosh (m-n) x]$,

$\sinh m x \cosh n x=\frac{1}{2}[\sinh (m+n) x+\sinh (m-n) x]$.

So $\mathscr{T}$ is a commutative and associative algebra.

Lemma 10. Let $\mathscr{T}$ be a linear space generated by $\{\sinh m x$, $\cosh n x \mid m, n \in \mathbf{N}\}$ over $\mathbf{F}$, and then $\{1, \sinh m x, \cosh n x \mid$ $m, n \in \mathbf{N}_{\mathbf{0}}$ \} is a basis of $\mathscr{T}$.
Proof. For $\forall n \in \mathbf{N}_{\mathbf{0}}$, suppose that there are $c_{0}, a_{i}, b_{j} \in \mathbf{F}, i, j \in$ $\mathrm{N}_{\mathbf{0}}$ such that

$$
\begin{aligned}
& c_{0}+a_{1} \sinh x+b_{1} \cosh x+\cdots+a_{n} \sinh n x+b_{n} \cosh n x \\
& =0 .
\end{aligned}
$$

We take derivative for (20) such that its derivative order is $2 k-1\left(k \in \mathbf{N}_{0}\right)$, and put $x=0$. Then we have

$$
a_{1}+2^{2 k-1} a_{2}+\cdots+n^{2 k-1} a_{n}=0 .
$$

Let $k=1,2, \ldots, n$, and then we obtain the following system of $n$ linear equations:

$$
\begin{gathered}
a_{1}+2 a_{2}+\cdots+n a_{n}=0 \\
a_{1}+2^{3} a_{2}+\cdots+n^{3} a_{n}=0 \\
\vdots \\
a_{1}+2^{2 n-1} a_{2}+\cdots+n^{2 n-1} a_{n}=0 .
\end{gathered}
$$

If $a_{1}, \ldots, a_{n}$ are seen to be unknown, then the coefficient matrix of (22) is the Vandermonde matrix whose determinant is not 0 , so $a_{i}=0, i=1, \ldots, n$.

We take derivative for (20) such that its derivative order is $2 k\left(k \in \mathbf{N}_{0}\right)$, and put $x=0$. Then we have

$$
b_{1}+2^{2 k} b_{2}+\cdots+n^{2 k} b_{n}=0 .
$$

Let $k=1,2, \ldots, n$, and then we obtain the following system of $n$ linear equations:

$$
\begin{gathered}
b_{1}+2^{2} b_{2}+\cdots+n^{2} b_{n}=0 \\
b_{1}+2^{4} b_{2}+\cdots+n^{4} b_{n}=0 \\
\vdots \\
b_{1}+2^{2 n} b_{2}+\cdots+n^{2 n} b_{n}=0 .
\end{gathered}
$$

If $b_{1}, \ldots, b_{n}$ are seen to be unknown, then the coefficient matrix of (24) is the Vandermonde matrix whose determinant is not 0 , so $b_{i}=0, i=1, \ldots, n$. Since, for any $i \in \mathbf{N}_{0}$, $a_{i}=0$ and $b_{i}=0$ satisfy (20), we have $c_{0}=0$. Hence $\{1$, $\sinh x, \cosh x, \ldots, \sinh n x, \cosh n x\}$ are linearly independent for any $n \in \mathbf{N}_{0}$, and then $\left\{1, \sinh n x, \cosh m x \mid n, m \in \mathbf{N}_{\mathbf{0}}\right\}$ are linearly independent and so they form a basis of $\mathscr{T}$ as desired.

Theorem 11. Let $\mathscr{A}_{1}, \mathscr{A}_{2}$ be commutative and associative algebras over $\mathbf{F}$. If $\varphi: \mathscr{A}_{1} \rightarrow \mathscr{A}_{2}$ is an isomorphism and $D_{1} \in \operatorname{Der} \mathscr{A}_{1}$, then the following statements hold:

(1) $D_{2}:=\varphi D_{1} \varphi^{-1} \in \operatorname{Der} \mathscr{A}_{2}$,

(2) $\varphi:\left(\mathscr{A}_{1}, D_{1}, \circ\right) \rightarrow\left(\mathscr{A}_{2}, D_{2}, \circ\right)$ is also an isomorphism of Novikov algebras. 
Proof. (1) For any $a, b \in \mathscr{A}_{1}$, we have

$$
\begin{aligned}
& \left(\varphi D_{1} \varphi^{-1}\right)(\varphi(a) \varphi(b)) \\
& \quad=\left(\varphi D_{1} \varphi^{-1}\right)(\varphi(a b)) \\
& \quad=\varphi D_{1}(a b)=\varphi\left(D_{1}(a) b+a D_{1}(b)\right) \\
& \quad=\varphi\left(D_{1}(a)\right) \varphi(b)+\varphi(a) \varphi\left(D_{1}(b)\right) \\
& \quad=\left(\varphi D_{1} \varphi^{-1}\right)(\varphi(a)) \varphi(b)+\varphi(a)\left(\varphi D_{1} \varphi^{-1}\right)(\varphi(b))
\end{aligned}
$$

So (1) holds.

(2) For any $a, b \in \mathscr{A}_{1}$, we have

$$
\begin{aligned}
\varphi(a \circ b) & =\varphi\left(a D_{1}(b)\right)=\varphi(a) \varphi\left(D_{1}(b)\right) \\
& =\varphi(a)\left(\varphi D_{1} \varphi^{-1}\right)(\varphi(b))=\varphi(a) D_{2}(\varphi(b)) \\
& =\varphi(a) \circ \varphi(b) .
\end{aligned}
$$

So (2) holds.

Theorem 12. Let $\mathscr{A}$ be a commutative and associative algebra over $\mathbf{F}$ satisfying Lemma 4 , let $D_{0}$ be its derivation satisfying (10), and let $\mathscr{T}$ be a commutative and associative algebra over F satisfying Lemmas 9 and 10 . If $\varphi: \mathscr{A} \rightarrow \mathscr{T}$ satisfies

$$
\begin{gathered}
\varphi\left(b_{m}\right)=\cosh m x, \quad m=0,1,2, \ldots, \\
\varphi\left(a_{n}\right)=\sinh n x, \quad n=1,2, \ldots,
\end{gathered}
$$

then the following statements hold:

(1) $\varphi$ is an isomorphism of commutative and associative algebras,

(2) $\varphi D_{0} \varphi^{-1}=\mathrm{d} / \mathrm{d} x$,

(3) $\varphi:\left(\mathscr{A}, a D_{0}, \circ\right) \rightarrow(\mathscr{T}, \varphi(a)(\mathrm{d} / \mathrm{d} x), \circ)$ is an isomorphism of Novikov algebras.

Proof. It is clear by Lemma 10, (8), and (19).

(2) By Lemma 6, we have

$$
\begin{aligned}
\varphi D_{0} \varphi^{-1}(\sinh n x) & =\varphi D_{0}\left(a_{n}\right) \\
& =\varphi\left(n b_{n}\right)=n \cosh n x \\
& =\frac{\mathrm{d} \sinh n x}{\mathrm{~d} x}, \\
\varphi D_{0} \varphi^{-1}(\cosh n x) & =\varphi D_{0}\left(b_{n}\right) \\
& =\varphi\left(n a_{n}\right)=n \sinh n x \\
& =\frac{\mathrm{d} \cosh n x}{\mathrm{~d} x} .
\end{aligned}
$$

So (2) holds.
(3) It is clear that $\varphi\left(a D_{0}\right) \varphi^{-1}=\varphi(a) \mathrm{d} / \mathrm{d} x$. By (27) and (10), we have

$$
\begin{aligned}
\varphi\left(a D_{0}\right) \varphi^{-1}(\sinh n x) & =\varphi\left(a D_{0}\right)\left(a_{n}\right) \\
& =\varphi\left(a D_{0}\left(a_{n}\right)\right)=\varphi\left(a n b_{n}\right) \\
& =\varphi(a) \varphi\left(n b_{n}\right)=\varphi(a) n \cosh n x \\
& =\frac{\varphi(a) \mathrm{d}(\sinh n x)}{\mathrm{d} x} .
\end{aligned}
$$

Similarly, we have $\varphi\left(a D_{0}\right) \varphi^{-1}(\cosh n x)=\varphi(a) \mathrm{d}(\cosh n x) /$ $\mathrm{d} x$. So $\varphi\left(a D_{0}\right) \varphi^{-1}=\varphi(a) \mathrm{d} / \mathrm{d} x$.

By Theorems 7 and 11 and Remark 3(2), we have

$$
\begin{aligned}
\varphi(b \circ c) & =\varphi\left(b a D_{0}(c)\right) \\
& =\varphi(b) \varphi\left(a D_{0}(c)\right) \\
& =\varphi(b)\left[\varphi\left(a D_{0}\right) \varphi^{-1}(\varphi(c))\right] \\
& =\frac{\varphi(b) \varphi(a) \mathrm{d}}{\mathrm{d} x(\varphi(c))} \\
& =\varphi(b) \circ \varphi(c), \quad \forall b, c \in \mathscr{A} .
\end{aligned}
$$

So $\varphi:\left(\mathscr{A}_{0}, a D_{0}, \circ\right) \rightarrow(\mathscr{T}, \varphi(a)(\mathrm{d} / \mathrm{d} x), \circ)$ is an isomorphism of Novikov algebras.

\section{Acknowledgments}

The authors would like to thank the referee for valuable comments and suggestions on this paper. This paper supported by NNSF of China (no. 11171055), NSF of Jilin province (No. 201115006), Scientific Research Foundation for Returned Scholars Ministry of Education of China, and the Fundamental Research Funds for the Central Universities.

\section{References}

[1] I. M. Gel'fand and L. A. Diki, "Asymptotic properties of the resolvent of Sturm-Liouville equations, and the algebra of Korteweg-de Vries equations," Functional Analysis and Its Applications, vol. 30, pp. 77-113, 1975.

[2] I. M. Gel'fand and L. A. Diki, "A Lie algebra structure in the formal calculus of variations," Functional Analysis and Its Applications, vol. 10, pp. 16-22, 1976.

[3] I. M. Gel'fand and I. Y. Dorfman, "Hamiltonian operators and algebraic structures associated with them," Functional Analysis and Its Applications, vol. 13, pp. 248-262, 1979.

[4] W. X. Ma, "Some Hamiltonian operators in infinite-dimensional Hamiltonian systems," Acta Mathematicae Applicatae Sinica, vol. 13, no. 4, pp. 484-496, 1990.

[5] W. X. Ma, "Complexiton solutions to the Korteweg-de Vries equation," Physics Letters A, vol. 301, no. 1-2, pp. 35-44, 2002.

[6] G. Z. Tu and W. X. Ma, "An algebraic approach for extending Hamiltonian operators," Journal of Partial Differential Equations, vol. 5, no. 1, pp. 43-56, 1992. 
[7] E. I. Zel'manov, "A class of local translation-invariant Lie algebras," Soviet Mathematics-Doklady, vol. 35, no. 1, pp. 216218, 1987.

[8] J. M. Osborn, "Simple Novikov algebras with an idempotent," Communications in Algebra, vol. 20, no. 9, pp. 2729-2753, 1992.

[9] J. M. Osborn, "Infinite-dimensional Novikov algebras of characteristic 0," Journal of Algebra, vol. 167, no. 1, pp. 146-167, 1994.

[10] X. Xu, "Hamiltonian operators and associative algebras with a derivation," Letters in Mathematical Physics, vol. 33, no. 1, pp. 1-6, 1995.

[11] X. Xu, "On simple Novikov algebras and their irreducible modules," Journal of Algebra, vol. 185, no. 3, pp. 905-934, 1996.

[12] X. Xu, "Novikov-Poisson algebras," Journal of Algebra, vol. 190, no. 2, pp. 253-279, 1997.

[13] X. Xu, "Variational calculus of supervariables and related algebraic structures," Journal of Algebra, vol. 223, no. 2, pp. 396-437, 2000.

[14] C. Bai and D. Meng, "The realization of non-transitive Novikov algebras," Journal of Physics A, vol. 34, no. 33, pp. 6435-6442, 2001.

[15] C. Bai and D. Meng, "A Lie algebraic approach to Novikov algebras," Journal of Geometry and Physics, vol. 45, no. 1-2, pp. 218230, 2003.

[16] C. Bai and D. Meng, "On the Novikov algebra structures adapted to the automorphism structure of a Lie group," Journal of Geometry and Physics, vol. 45, no. 1-2, pp. 105-115, 2003.

[17] L. Chen, Y. Niu, and D. Meng, "Two kinds of Novikov algebras and their realizations," Journal of Pure and Applied Algebra, vol. 212, no. 4, pp. 902-909, 2008.

[18] Y. Kang and Z. Chen, "Novikov superalgebras in low dimensions," Journal of Nonlinear Mathematical Physics, vol. 16, no. 3, pp. 251-257, 2009.

[19] F. Zhu and Z. Chen, "Novikov superalgebras with $A_{0}=A_{1} A_{1}$," Czechoslovak Mathematical Journal, vol. 60(135), no. 4, pp. 903907, 2010. 


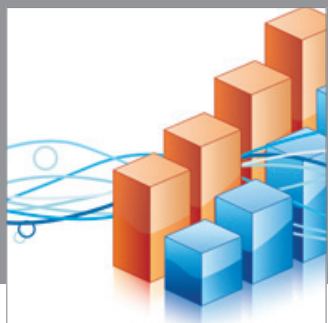

Advances in

Operations Research

mansans

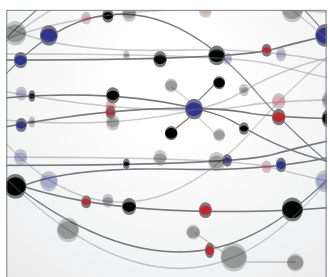

The Scientific World Journal
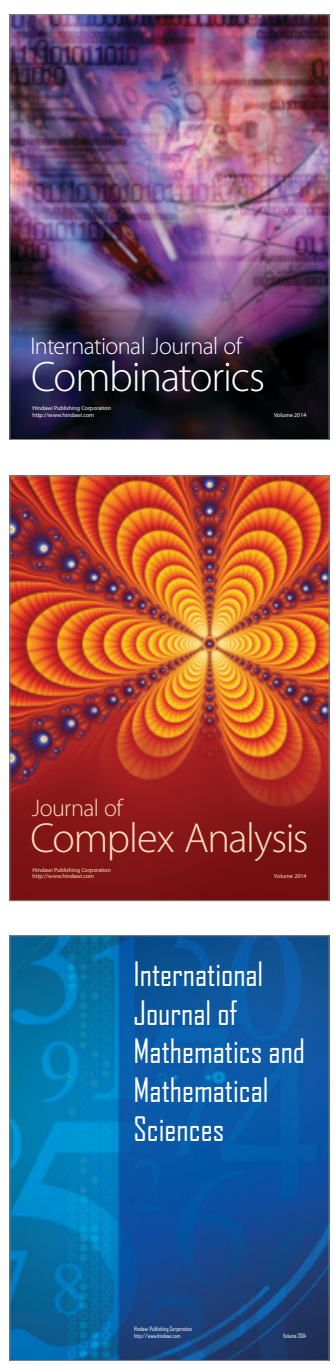
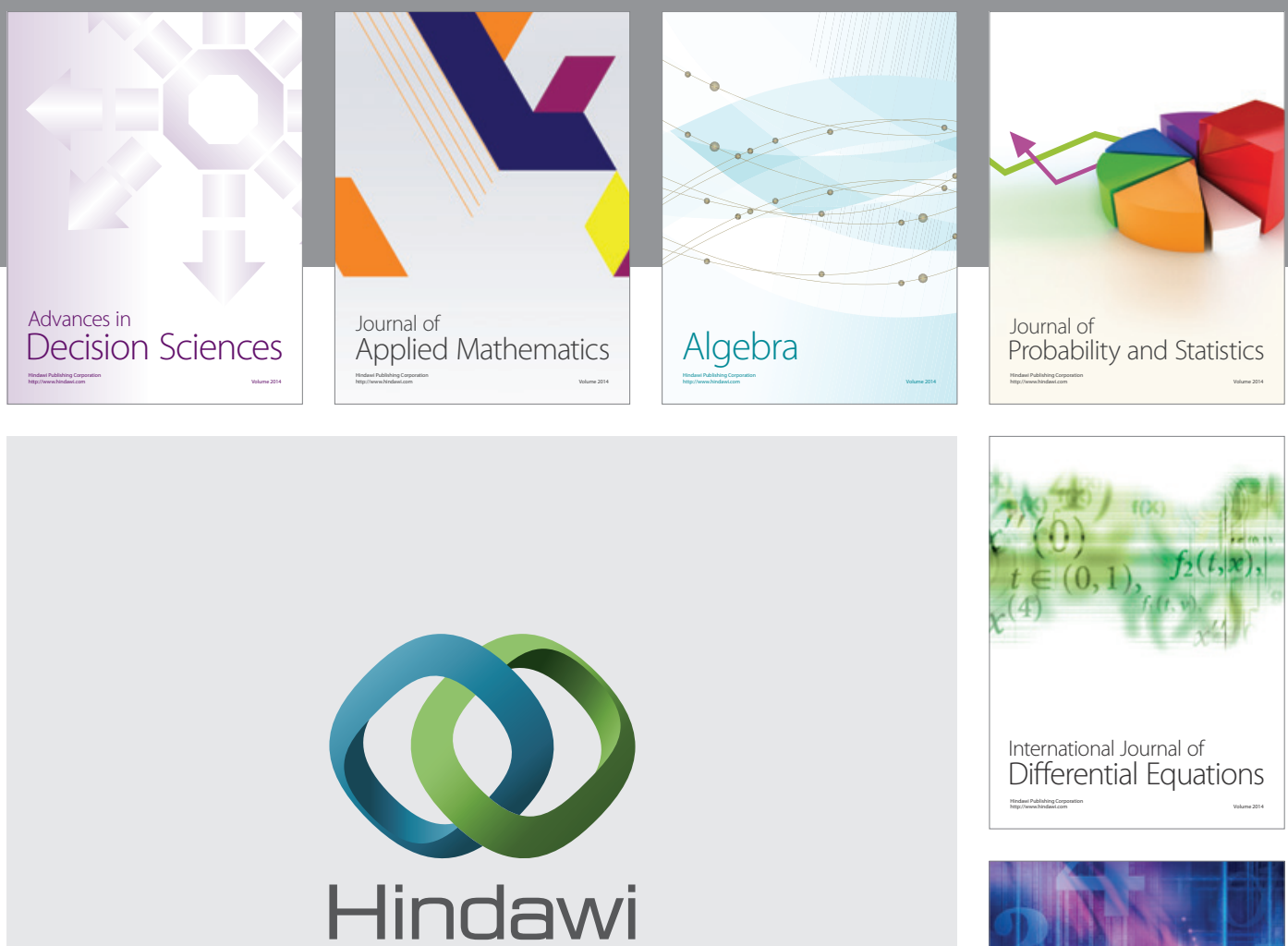

Submit your manuscripts at http://www.hindawi.com
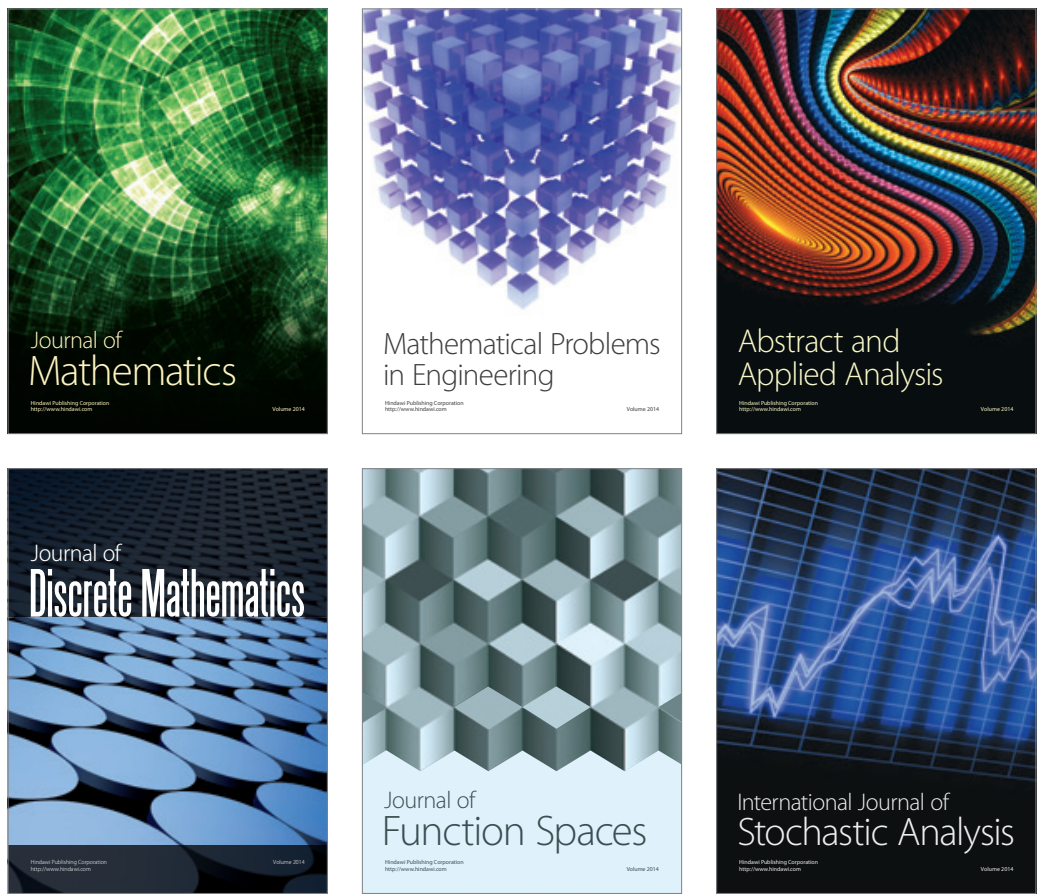

Journal of

Function Spaces

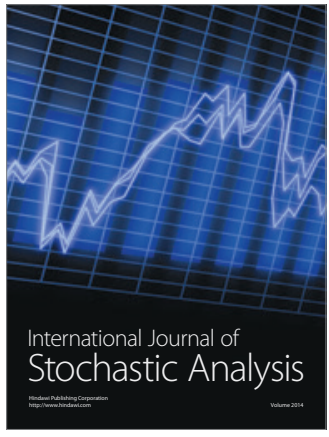

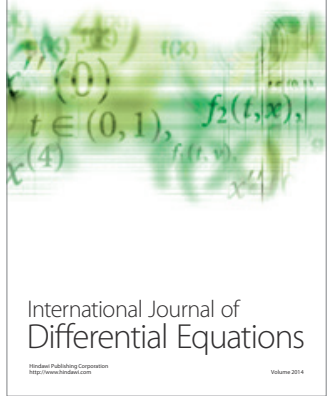
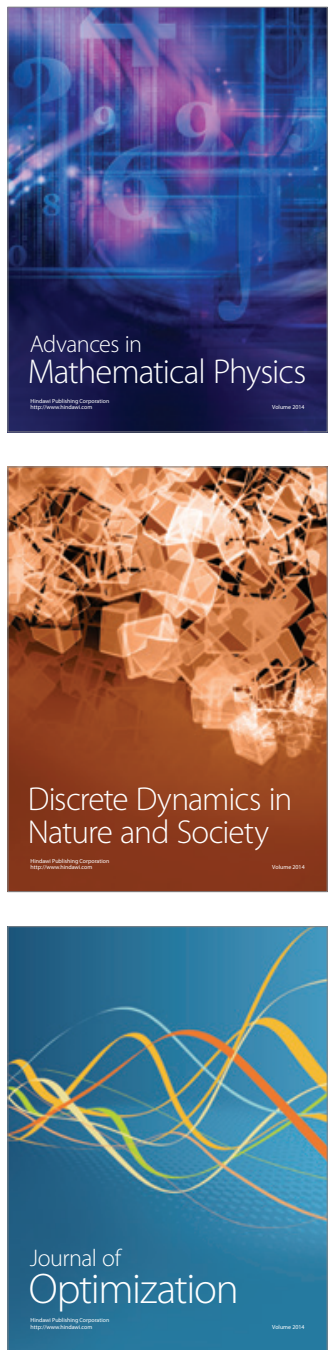